\title{
Spectral and temporal control of a random Erbium doped fiber laser
}

\author{
Can Yao, Camille Sophie Brès, Luc Thévenaz \\ Ecole Polytechnique Fédérale de Lausanne (EPFL), Photonic Systems Laboratory (PHOSL), STI-IEL, Station 11, CH-1015 Lausanne, \\ Switzerland \\ Author e-mail address: can.yao@epfl.ch
}

\begin{abstract}
A random Q-switched Erbium fiber laser is obtained with an average repetition rate to several kilohertz, and its output spectrum can be easily flipped from 1535 to $1550 \mathrm{~nm}$ by a fiber loop mirror. (C) 2018 The Author(s)

OCIS codes: (140.0140) Lasers and laser optics; (230.0230) Optical devices
\end{abstract}

\begin{abstract}
1. Introduction
Random laser has attracted much research interest for decades, due to its properties such as low coherence and high brightness, which is very promising for applications like speckle free imaging [1]. Wherever, random laser in traditional bulk materials such as crystal powder, colloidal quantum dot and liquid crystal, et, al. [2][3], usually exhibits poor lasing directionality and control. In this decade, random fiber laser has been investigated tremendously since its invention in 2010 [4], and its properties such as cavity-free, directionality, high intensity, mode-free, long distance and thermal stability, have made it a promising tool in optical sensing, optical communication, and random number generation.

The feedback of random fiber laser is provided by Rayleigh scattering in the optical fiber. Thus, the pulsing operation of random fiber lasers is challenging as the feedback is random and hard to control. However, researchers have found that the stimulated Brillouin scattering will induce pulse generation in random fiber laser [5]-[8]. It is always interesting to find an easy method to generate pulsed random fiber lasing. Here we present our preliminary experimental results of a random Q switched Erbium doped fiber laser.

Besides, the spectrum control of the Erbium doped random fiber laser has been reported, while it is always interesting to find an easy method to control the output spectrum of the random fiber laser [9], [10]. Here we also present the spectrum control of the Erbium doped random fiber laser simply by tuning the polarization in a fiber loop mirror.
\end{abstract}

\section{Experiment results and discussion}

The experimental set-up of the proposed Erbium doped random fiber is shown in Figure 1. A $980 \mathrm{~nm}$ laser diode with maximum power around $138 \mathrm{~mW}$ is used to pump the EDF in the forward direction, through a 980-/1550-nm wavelength-division multiplexer (WDM). The EDF is a $100 \mathrm{~m}$ long fiber with light Erbium doping concentration of around $50 \mathrm{ppm}$, and heavy GeO2 doping concentration of around $12.94 \mathrm{wt} \%$. The EDF has the peak absorption coefficient of $0.15 \mathrm{~dB} / \mathrm{m}$ at $1532 \mathrm{~nm}$. The $1550 \mathrm{~nm}$ port of the WDM was connected with a FLM, composing a $3 \mathrm{~dB}$ coupler and a polarization controller (PC). The reflection of the FLM is measured between $11 \%$ and $65 \%$. An optical spectrum analyser (OSA) with spectrum resolution down to $0.02 \mathrm{~nm}$ was used to measure the laser spectrum output. An oscilloscope is used to measure the temporal behavior of the random fiber laser. A $4 \mathrm{~km}$ DCF was placed after the $\mathrm{EDF}$, and the total loss of the DCF at $1550 \mathrm{~nm}$ is about $3.5 \mathrm{~dB}$. An isolator was placed after the DCF to eliminate the unwanted Fresnel reflection.

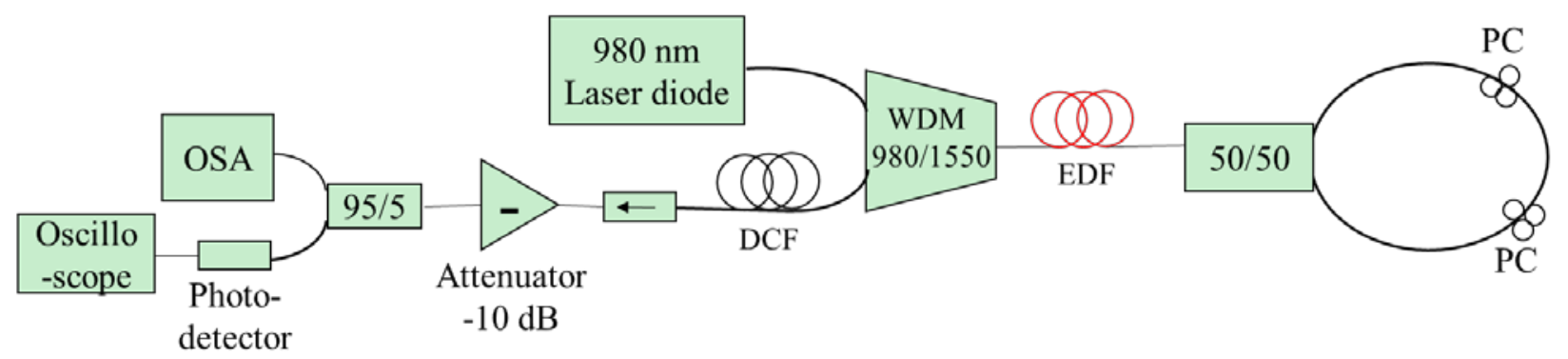

Figure 1. Experimental set-up of the Erbium doped random fiber laser. 
The oscilloscope detects the temporal behavior of the Erbium doped random fiber laser as shown in Figure 2 (a), indicating random Q-switched pulsing generation, and the average repetition rate increases with the pump power (Figure 2 (b)) from hundreds of $\mathrm{Hz}$ to several thousand $\mathrm{Hz}$. However, here we are quite limited by the pump power, and it is likely we are only at the initial stage of random Q-switching in the random fiber laser [11].
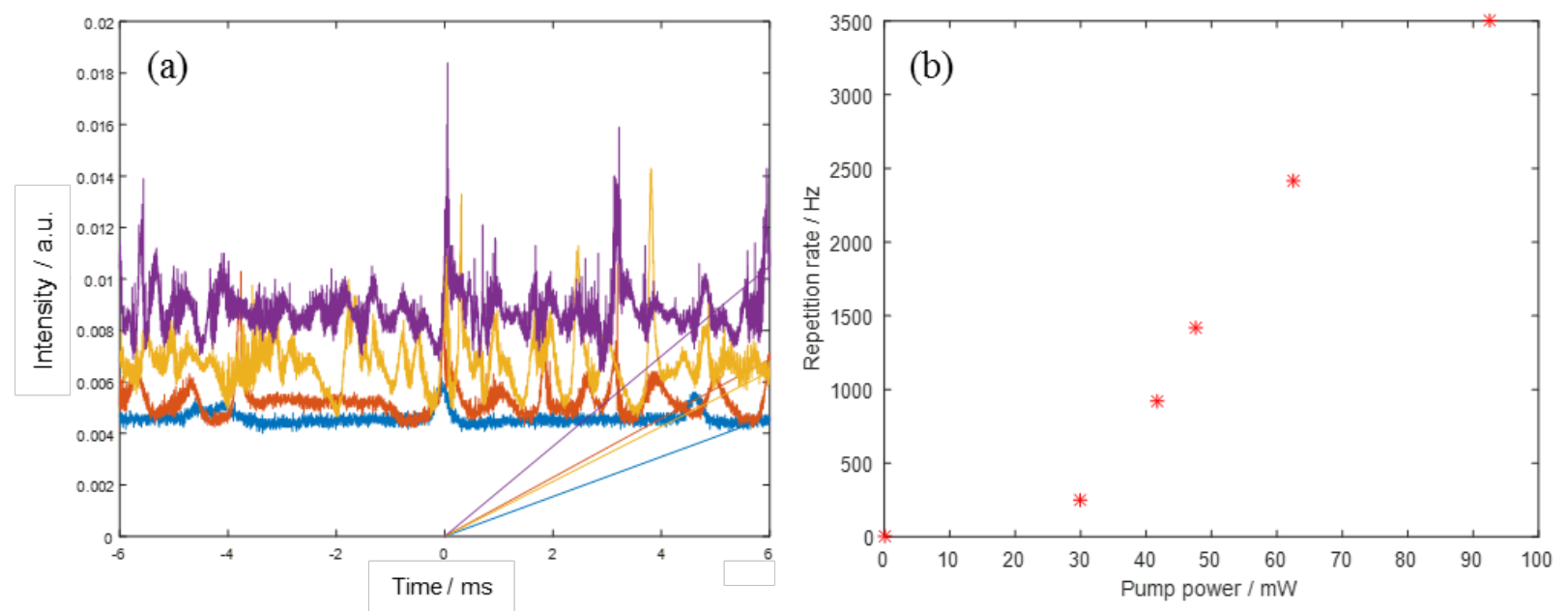

Figure 2. (a) Temporal spectrum of the random fiber laser at different pump power. Blue-30 mW. Orange-41.6 mW. Yellow-62.57 $\mathrm{mW}$. Purple-92.6 mW. (b) The repetition rate increases with the pump power.

Besides, as we have previously shown that, the spectrum output of the Erbium doped random fiber laser can also be flipped between the range around $1535 \mathrm{~nm}$ and $1550 \mathrm{~nm}$, simply by flipping the polarization controller in the fiber loop mirror [12]. This is due to the severe gain competition between these two Erbium emission peaks. Since the polarization has a random relation with the random fiber laser output spectrum, it is believed that a fast and regular polarization control can be applied here for random number generation while set the output at wavelength $1535 \mathrm{~nm}$ as "0" and at $1550 \mathrm{~nm}$ as " 1 ”, or vice versa.

\section{Conclusions}

In conclusion, we have presented a simple method to obtain random Q-switched Erbium doped fiber laser for incoherent pulse generation. Further study can be outlooked here, for example, to insert a saturable absorber in the set-up and check its effect on the random Q-switching. Besides, based on that the spectrum output of the Erbium doped random fiber laser can also be flipped between the range around $1535 \mathrm{~nm}$ and $1550 \mathrm{~nm}$, simply by a polarization controller in the fiber loop mirror, we expect this set-up can be applied for simple random number generation.

[1] H. Cao, "Review on latest developments in random lasers with coherent feedback," J. Phys. A. Math. Gen., vol. 38, pp. 10497-10535, Dec. 2005.

[2] C. J. S. De Matos, L. D. S. Menezes, A. M. Brito-silva, and M. A. Martinez, "Random Fiber Laser,” Phys. Rev. Lett., vol. 99, no. 153903, 2007.

[3] Y. Nagai, R. Fujimura, and K. Kajikawa, “Coherent random laser fluid of nematic liquid crystal emulsions,” Jpn. J. Appl. Phys., vol. 53, no. 1 PART 2, 2014.

[4] S. K. Turitsyn, S. A. Babin, A. E. El-taher, P. Harper, D. V Churkin, V. Karalekas, E. V Podivilov, S. I. Kablukov, and J. D. Aniacastan, "Random distributed feedback fibre laser," Nat. Photonics, vol. 4, no. April, pp. 231-235, 2010.

[5] M. Fan, Z. Wang, H. Wu, W. Sun, and L. Zhang, "Low-Threshold, High-Efficiency Random Fiber Laser With Linear Output,” IEEE Photonics Technol. Lett., vol. 27, no. 3, pp. 319-322, 2015.

[6] J. Lee, J. Koo, P. Debnath, Y.-W. Song, and J. H. Lee, "A Q -switched, mode-locked fiber laser using a graphene oxide-based polarization sensitive saturable absorber,” Laser Phys. Lett., vol. 10, no. 3, p. 35103, Mar. 2013.

[7] R. Ma, W. L. Zhang, X. P. Zeng, Z. J. Yang, Y. J. Rao, B. C. Yao, C. B. Yu, Y. Wu, and S. F. Yu, "Quasi mode-locking of coherent feedback random fiber laser," Sci. Rep., vol. 6, no. December, p. 39703, 2016.

[8] Y. Tang and J. Xu, “A random Q-switched fiber laser,” Sci. Rep., vol. 5, p. 9338, 2015.

[9] S. Saleh, N. A. Cholan, A. H. Sulaiman, and M. A. Mahdi, “Stable Multiwavelength Erbium-Doped Random Fiber Laser,” IEEE J. Sel. Top. Quantum Electron., vol. 24, no. 3, pp. 3-8, 2017.

[10] L. Wang, X. Dong, P. Shum, and H. Su, “Tunable erbium-doped fiber laser based on random distributed feedback,” IEEE Photonics J., vol. 655, no. c, pp. 1-1, 2014.

[11] S. Wang, W. Lin, W. Chen, C. Li, C. Yang, T. Qiao, and Z. Yang, "Low-threshold and multi-wavelength Q-switched random erbiumdoped fiber laser," pp. 0-4, 2016.

[12] C. Yao, L. Thevenaz, and C. S. Bres, "Erbium doped random fiber laser and fiber mixing effect,” Int. Conf. Transparent Opt. Networks, pp. 12-15, 2017. 